\title{
BMJ Open Remotely delivered environmental enrichment intervention for traumatic brain injury: Study protocol for a randomised controlled trial
}

\author{
Zorry Belchev (D) ,,2,3 Mary Ellene Boulos (D) ,3,4 Julia Rybkina (D) ,3,4 \\ Kadeen Johns, ${ }^{3}$ Eliyas Jeffay, ${ }^{1,2,3}$ Brenda Colella, ${ }^{3}$ Jason Ozubko, ${ }^{5}$ \\ Michael Johnathan Charles Bray (1) , Nicholas Di Genova, ${ }^{3,6}$ Adina Levi, ${ }^{2,7}$ \\ Alana Changoor, ${ }^{3,8}$ Thomas Worthington, ${ }^{3,7}$ Asaf Gilboa, ${ }^{1,2,3}$ Robin Green ${ }^{3,9}$
}

To cite: Belchev Z, Boulos ME, Rybkina J, et al. Remotely delivered environmental enrichment intervention for traumatic brain injury: Study protocol for a randomised controlled trial. BMJ Open 2021;11:e039767. doi:10.1136/ bmjopen-2020-039767

- Prepublication history and additional material for this paper are available online. To view these files, please visit the journal online (http://dx.doi. org/10.1136/bmjopen-2020039767).

$A G$ and $R G$ contributed equally.

Received 27 April 2020

Revised 24 October 2020

Accepted 27 November 2020

Check for updates

(C) Author(s) (or their employer(s)) 2021. Re-use permitted under CC BY-NC. No commercial re-use. See rights and permissions. Published by BMJ.

For numbered affiliations see end of article.

Correspondence to

Ms Zorry Belchev;

zorrybelchev@gmail.com

\section{ABSTRACT}

Introduction Individuals with moderate-severe traumatic brain injury (m-sTBI) experience progressive brain and behavioural declines in the chronic stages of injury. Longitudinal studies found that a majority of patients with m-sTBI exhibit significant hippocampal atrophy from 5 to 12 months post-injury, associated with decreased cognitive environmental enrichment (EE). Encouragingly, engaging in EE has been shown to lead to neural improvements, suggesting it is a promising avenue for offsetting hippocampal neurodegeneration in m-sTBI. Allocentric spatial navigation (ie, flexible, bird's eye view approach), is a good candidate for EE in m-sTBI because it is associated with hippocampal activation and reduced ageing-related volume loss. Efficacy of EE requires intensive daily training, prohibitive within most current health delivery systems. The present protocol is a novel, remotely delivered and self-administered intervention designed to harness principles from $\mathrm{EE}$ and allocentric spatial navigation to offset hippocampal atrophy and potentially improve hippocampal functions such as navigation and memory for patients with m-sTBI. Methods and analysis Eighty-four participants with chronic $\mathrm{m}$-sTBI are being recruited from an urban rehabilitation hospital and randomised into a 16-week intervention (5 hours/week; total: 80 hours) of either targeted spatial navigation or an active control group. The spatial navigation group engages in structured exploration of different cities using Google Street View that includes daily navigation challenges. The active control group watches and answers subjective questions about educational videos. Following a brief orientation, participants remotely self-administer the intervention on their home computer. In addition to feasibility and compliance measures, clinical and experimental cognitive measures as well as MRI scan data are collected pre-intervention and post-intervention to determine behavioural and neural efficacy.

Ethics and dissemination Ethics approval has been obtained from ethics boards at the University Health Network and University of Toronto. Findings will be presented at academic conferences and submitted to peer-reviewed journals.

\section{Strengths and limitations of this study}

- A remote, self-administered intervention will allow patients greater access to clinically relevant resources regardless of physical and economical restrictions, and increase current telerehabilitation offerings.

- The inclusion of neuroimaging outcomes allow for the examination of structural changes that may occur alongside potential cognitive improvements associated with targeted behavioural training, increasing our understanding of the mechanisms of these potential changes in humans.

- The inclusion of an active control group is critical in determining whether targeted rather than generalised training is effective in improving hippocampaldependent abilities, but introduces a conservative bias in observing these effects, more so than if only a waitlisted control group was included.

- Although the present protocol builds on the principles of successful environmental enrichment observed in rodents in terms of targeted training and high dose, a potential component that may mediate the benefit is the addition of vigorous physical exercise, which will be a consideration for future studies.

Trial registration number Version 3, ClinicalTrials.gov Registry (NCT04331392).

\section{INTRODUCTION}

Traumatic brain injury (TBI) is a significant global public health problem, with an estimated worldwide incidence rate of 874-1005 cases per $100000 .{ }^{1}$ Contrary to typical models of the recovery trajectory, recent longitudinal studies show that degeneration continues at least up to 2 years postinjury. ${ }^{2-4}$ These recent lines of evidence have signalled a shift from viewing TBI as a neurologically stable disorder in the subacute and chronic stages, to treating it as a chronic and 
neurodegenerative disorder, ${ }^{5}$ necessitating a need for appropriate long-term treatments for patients past their acute phase of recovery.

\section{Atrophy and cognitive impairment in chronic TBI}

There is evidence that moderate-severe TBI (m-sTBI) results in chronic progressive volumetric decline in both white matter and grey matter through the first and up to at least the second year post-injury. Structures affected include the fornix, ${ }^{7}$ corpus callosum, ${ }^{3}$ and temporal, frontal and occipital regions. ${ }^{2}$ Notably, the hippocampus (HPC) is vulnerable to chronic degeneration, where Green $e t a l^{3}$ found over $70 \%$ of patients showed significant declines (over 2 SDs away from controls) in the HPC. In another study, specific degeneration was found in the HPC, in addition to subcortical regions that included the thalamus, putamen, amygdala and caudate. ${ }^{2}$ The vulnerability of the HPC has been attributed to its sensitivity to excitotoxicity ${ }^{8}$ and Wallerian degeneration through damage distal to the site of the injury. ${ }^{9-11}$

In addition to neural declines, patients with TBI also exhibit chronic cognitive deficits, including in spatial abilities $^{12}$ and memory. ${ }^{13} 14$ These types of cognitive deficits have been linked to atrophy in the HPC. ${ }^{1516}$ Furthermore, these cognitive deficits may generalise to daily functioning, as lower acute volumes in areas known to be associated with cognitive deficits in TBI (ie, HPC, thalamus) predict poorer functional outcome measured in the chronic stage. ${ }^{17}$

\section{Impact of interacting factors on recovery and decline}

An interaction between certain factors can also negatively contribute to continued degeneration in the chronic stages of TBI by adding to or compounding persistent underlying neuropathology. ${ }^{18} 19$ As outlined by the negative neuroplasticity framework, ${ }^{20}{ }^{21}$ the following key factors can interact and have a negative impact on recovery from a TBI by driving negative neuroplasticity, including: (1) reduced schedules of activity following rehabilitation regimen in the acute periods; (2) noisy processing from sensory deficits; (3) weakened neuromodulatory control due to neurotransmitter dysfunction; and (4) the combination of the preceding factors culminating in negative learning through preference for lowlevel, low-effort cognitive tasks. Thus, the chronic period that follows in-patient rehabilitative treatments is critical for ensuring continued cognitive and neural stimulation, with therapeutic support during this period that can be scalable and does not necessitate extensive therapist involvement.

\section{Harnessing environmental enrichment to improve post-injury outcomes}

The HPC is particularly vulnerable to TBI, ${ }^{2-4} 17$ yet also holds an innate affinity for neurogenesis and neuroplasticity, ${ }^{22-24}$ highlighting it as a good candidate for targeted interventions. Evidence from animal studies shows that environmental enrichment (EE) through extensive maze training can support positive neuroplasticity in the HPC. ${ }^{25}$ Support for this intervention approach has also been shown in humans, particularly when focusing on allocentric spatial navigation, involving flexible navigation from a bird's eye view perspective. ${ }^{26}{ }^{27}$ For example, an intensive 90 hours of training on a video game associated with allocentric navigation resulted in increased HPC volume in those who generally use the opposite strategy (ie, egocentric). ${ }^{28}$ Another study showed that intensive virtual navigation training in healthy older adults resulted in the successful attenuation of expected age-related volume declines in the $\mathrm{HPC}^{29}$ and increased hippocampal neural density that was moderated by genotype. $^{30}$

Informed by the efficacy of such interventions, here we designed a novel intervention focused on three factors: (1) targeting the HPC through training on allocentric navigation; (2) high-intensity and high-dose schedules; and (3) scalability and convenience through remote training online. Google Street View (GSV) was chosen as the navigation platform because it is easily accessible from patients' homes and does not require specialised software, allowing intensive training of allocentric navigation with only a browser and internet. Based on current experimental evidence, we created a website with set virtual environments (cities) consisting of route learning and associated navigation tasks such as vector mapping, distance judgements, reverse and blocked routes, and landmark mapping known to significantly engage hippocampal function. The dose of the structured navigation training is 80 hours based on previous findings that produced significant benefits. ${ }^{29}$ Different levels of difficulty are available to adjust to patients' ability and maintain an appropriate challenge level. To ensure compliance, intrinsic and extrinsic rewards are embedded into the intervention, and remote progress tracking allows for immediate assistance.

\section{Study objectives and hypotheses}

The present protocol is a component of a program of research with three types of patient populations (m-sTBI, multiple sclerosis, patients with TBI living in remote areas). The present study examines the efficacy and feasibility of a novel, remotely delivered and self-administered intervention designed to offset HPC atrophy following $\mathrm{m}$-sTBI. The primary objective is to examine the impact of treatment on: (1a) spatial abilities and memory employing HPC-sensitive experimental behavioural measures; and (1b) chronic HPC volume loss employing quantitative MRI of the HPC. As compared to an active control condition that provides generalised $\mathrm{EE}$, we predict that the navigation intervention will lead to more HPC-specific neural and behavioural improvements. The secondary objective is to assess the feasibility of the intervention. For a broad overview of the trial registration data set, please refer to online supplemental table 1 . 


\section{METHODS}

\section{Participants}

Patients with m-sTBI from Toronto Rehabilitation Institute's Acquired Brain Injury Rehab Service in-patient and day hospital programmes who are under 7 months post-injury are in the process of being recruited, with a projected recruited sample size of $n=84$. This sample size accounts for an estimated attrition rate of just under $20 \%$, with an expected resultant sample size of $n=70$ (35 per group). When factoring in attrition, this target sample size will achieve 0.80 power to detect medium-large effects based on a power analysis for a linear regression with eight predictors (using $G^{*}$ Power $^{31}$ ). Power will be further maximised by employing dimension reduction methods (ie, principal component analysis) prior to conducting the linear regression analyses, with the projected number of resultant predictors not exceeding eight.

Inclusion criteria include: (1) acute care diagnosis of m-sTBI; (2) post-traumatic amnesia (PTA) of 24 hours or more and/or lowest Glasgow Coma Scale (GCS) $<13$; (3) positive CT or MRI; (4) between 18 and 55 years of age; (5) fluency in English; (6) competency to provide informed consent or availability of a legal substitute decision maker; (7) basic computer skills (use of internet/ email, mouse and arrow keys); (8) functional use of at least one upper extremity for computer use; and (9) resident of Greater Toronto Area (to facilitate access to the MRI). The age cut-off excluded patients over 55 years to prevent potential age-related confounds in brain volume and cognitive changes, as research has shown that decline begins to accelerate in the fifth to sixth decade of life. ${ }^{32}$ Exclusion criteria include: (1) neurological disorder other than TBI (eg, dementia, stroke); (2) diagnosis of a neurodevelopmental disorder; (3) TBI sustained before age 18 years; (4) systemic comorbidities (eg, lupus, diabetes); (5) current diagnosis of aphasia; and (6) presence of metal inside the body (eg, surgical clips, pacemaker) leading to ineligibility for an MRI. It is noted that although exclusion on the basis of a diagnosis of aphasia is necessary for establishing proof of principle, future development should emphasise the incorporation of aphasia-friendly materials in order to benefit as many patients as possible.

Inclusion criteria pertaining to the participant's medical history are assessed using a chart review. During recruitment, patients are informed that they do not need to discontinue ongoing rehabilitative activities to participate in the study. Basic computer skills are evaluated at the pre-intervention assessment.

Participants are compensated for their participation by receiving \$C75 following the completion of each of the pre-intervention and post-intervention assessments, and \$C75 in electronic gift cards (ie, Amazon) for each month of intervention completion. To maximise retention and compliance to intervention dose, participants also have the opportunity to receive an additional \$C40 in coffee cards ( $\$ 2.50$ for each completed week). A consideration regarding compensation is that though it is a necessary component of research studies, its inclusion may impact the assessment of the feasibility of implementing the intervention clinically, in terms of both uptake and compliance. This is partially addressed by a debriefing question posed to participants in a post-intervention semistructured interview, which asks whether they would have completed the intervention without compensation (for more detailed information, refer to the outcome measures and online supplemental table 2). Based on the hourly rate (\$C4.25), it is unlikely to provide significant financial incentive. Furthermore, once the efficacy of the intervention is validated, this would provide an inherent incentive for patients to complete it as part of their clinical rehabilitation and recovery.

\section{Patient and public involvement}

A recovered patient with TBI was involved in the initial conceptualization of the study. Once the intervention was developed, pilot patients were recruited to complete $3-5$ weeks of the designed intervention to gauge the feasibility of the format and intensity of remote training; the patients were able to complete the training as designed and showed improvements on the targeted training tasks. Patients in the present study are asked to assess the burden of the intervention through the weekly administration of the How Much Is Too Much Scale. ${ }^{33}$ As part of a post-intervention semistructured interview, they are also asked to identify any facilitators and barriers they encountered to completing the intervention, as well as the average amount of time they required to participate in the intervention. The public was not involved in the design of the study, but a number of public organisations are engaged for recruitment aid (eg, March of Dimes, local community brain injury services). Study contacts at each organisation are provided with a recruitment flyer for circulation among their case managers and in communication with their members (eg, monthly newsletters).

\section{Study design}

The present study is a randomised, controlled, patient and (partially) observer blinded, parallel group, two arm, superiority trial with a 1:1 allocation. Participants are randomly assigned to either the experimental targeted navigation intervention or to active control training (educational videos), and are blinded to their assigned experimental versus active control condition. Randomisation is conducted using the rand() function in Microsoft Excel by the study coordinator prior to study commencement. A central recruiter from the institutional recruitment office who is not part of the study team is employed, who engages in initial contact and primary screening. The study coordinator then conducts secondary screening and formal enrolment by assigning participants their study identity numbers, which are not directly linked to group allocation. The study team members conducting the in-person assessments, as well as the MRI technologist, are blinded to group assignments. The experimenter conducting the remote assessments and training is not 
CONSORT Study Flow Diagram

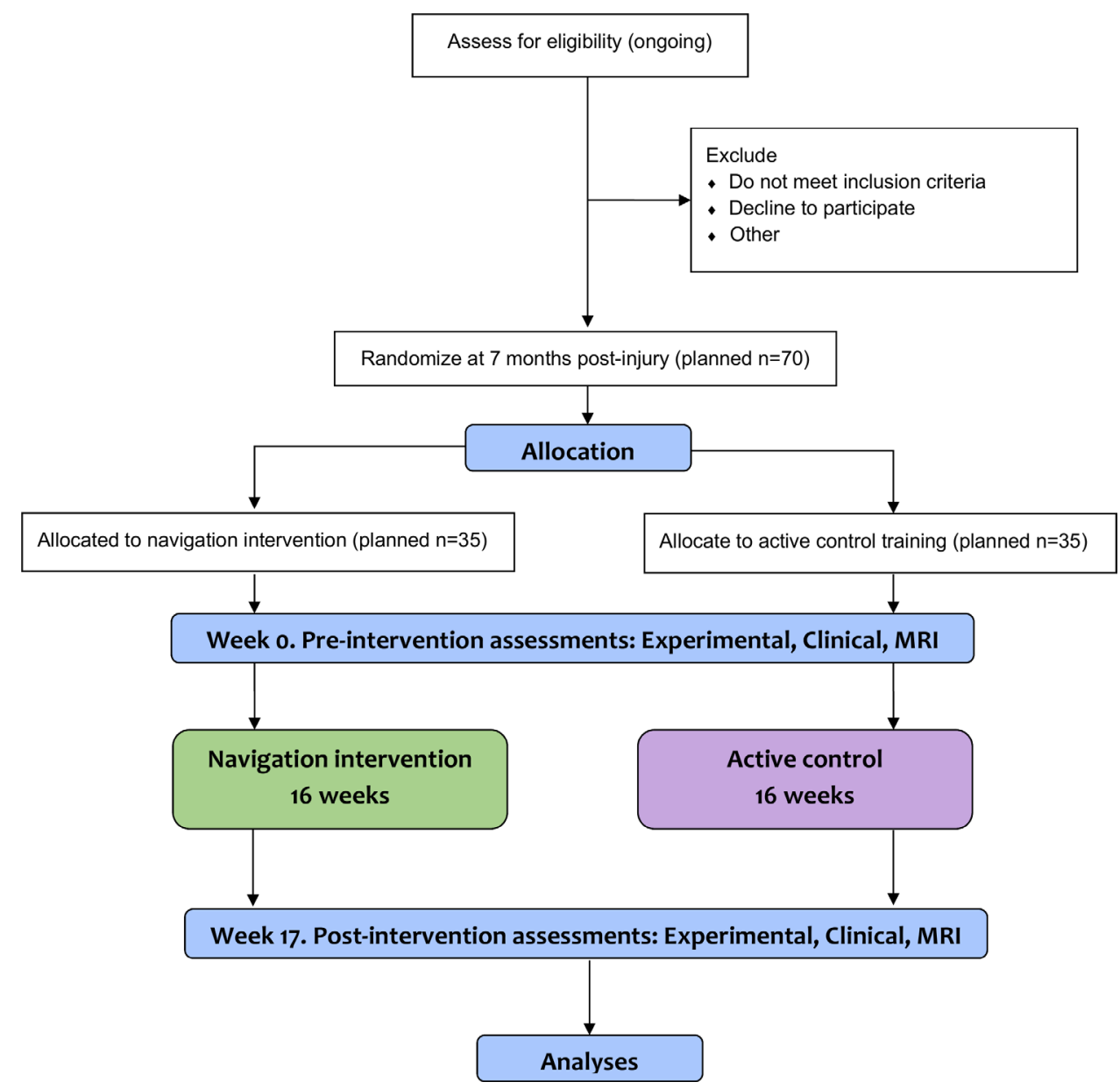

Figure 1 CONSORT study flow diagram of the present protocol. CONSORT, Consolidated Standards of Reporting Trials.

blinded to group assignments, as they need to train the participant on the task, but potential bias is minimised by the absence of non-verbal cues over the phone and close adherence to standardised scripts. Participants complete their assigned intervention online using their computer at home. In the event that a participant does not have access to a computer or internet connection, these are lent to them at no cost for the duration of the study.

Cognitive assessments are conducted at pre-intervention (week 0) and post-intervention (week 17) by the study team and are each split over 2 days (for a Consolidated Standards of Reporting Trials study flow diagram, see figure 1). Participants are scheduled to complete the pre-intervention assessment at approximately 7 months post-injury, in order for the intervention to occur during a time period over which hippocampal degeneration is generally observed. ${ }^{2-4}$ Participants who are recruited at the acute stage post-injury are put on a waitlist until they reach 7 months post-injury. Pre-intervention assessment occurs 1 week prior to beginning the intervention, in-person at the University of Toronto Neuroimaging Facility (for MRI), and over the phone. At the end of the pre-intervention assessments, participants receive orientation and training for their assigned intervention by completing a session on the respective intervention's website, guided by the experimenter over the phone. Participants assigned to the navigation intervention are provided with instructions on how to develop their allocentric navigation strategy in a manner that is conducive to flexible navigation and thereby linked to HPC activation. ${ }^{34} 35$

Reminder emails are sent out immediately following the orientation, and on day 1 of the intervention in order to support participants when they are beginning the intervention. For the remainder of the 16 -week intervention, participants receive reminder emails only if they miss 3 days of training, and a phone call if they miss an entire week of training, in order to work through any potential barriers to participation. Daily text-message or email reminders are added as needed. A month before the end of the intervention period, the post-intervention assessments are scheduled to ensure that they are conducted a maximum of 1-2weeks following intervention completion.

For an overview of the specific roles and responsibilities involved in conducting the study, refer to online supplemental table 3 . 


\section{Targeted navigation intervention protocol}

The targeted navigation intervention involves virtual navigation training with a focus on allocentric navigation. ${ }^{36} 37$ The dose and intensity match the design of a previous study with successful outcomes ${ }^{29}{ }^{30}$ : approximately 1 hour a day, 5 days a week, for 16 weeks. The intervention is delivered on a designated website hosted within the University Health Network's (UHN) secure servers. Each week, participants learn a section of a new city with basic to more challenging navigation tasks, with the goal of being able to independently navigate the assigned section by the end of the week. During protocol development, cities were selected based on availability in GSV, presence of English street names, sufficient size (eg, a city centre at least $1 \mathrm{~km}^{2}$ ), and availability of notable landmarks.

Participants are asked to complete tasks in new cities with increasingly challenging layouts each week. Prior to starting the intervention, participants are administered a survey of cities visited to ensure all cities are novel to participants. If a participant has visited a test city, a replacement city is assigned. The challenge levels of the tasks within each week vary based on the selected level, ranging from level 1 to 4 , which differ in number of streets, turns and landmarks (see below). All participants are initially placed in level 1 , and levels for subsequent weeks are based on performance of at least $80 \%$ on all of the main task measures during the previous week. If participants are performing less than $60 \%$ on any of the main task measures in subsequent weeks, they are placed in a lower level the following week. If participants are performing between $60 \%$ and $79 \%$ on any of the main task measures, they remain in the same level they were assigned the previous week.

Inspired by the format of the study maps used in another study, ${ }^{38}$ each week of training begins with studying a map stripped of all labelling except for preselected landmarks and street names. Based on the challenge level, an initial 3 landmarks and 8-10 street names were selected for levels 1-3, and an initial 5 landmarks and 11-13 street names were selected for level 4 . Throughout the week, participants learn additional landmarks and street names, where by the end of the week they will have learnt 5 landmarks and 11-13 street names for levels 1 and 2, 7 landmarks and 14-16 street names for level 3, and 9 landmarks and 17-20 street names for level 4.

Scaffolding learning is applied using three types of activities that increase in difficulty throughout the week, including: (1) navigation, which requires participants to navigate routes of increasing difficulty; (2) a set of endof-day multiple choice challenge questions, testing information learnt during the day and throughout the week; and (3) a map placement task to assess street and landmark place memory.

Navigation includes: (1) passive routes, where participants are shown videos of route navigation in GSV between each of the new landmarks; (2) active routes, which require participants to independently navigate the same routes shown in the videos; (3) reverse routes, which require participants to navigate to and from landmarks in the opposite direction from what was learnt in the videos; (4) alternate/new routes, which involve finding routes to learnt landmarks or intersections that had not been previously paired; and (5) blocked routes, where participants are instructed to find detours to landmarks or intersections when certain streets are identified as inaccessible. When navigating each route, participants are provided a dual-map view earlier in the week, with a map inset on one half of the screen, and GSV on the other half. Later in the week, participants are required to navigate solely using GSV. Dual-maps and GSV are embedded in a new page within the website using scripts incorporating Google Maps javascript API, which collects participant path data and confirms they have reached the target location.

The second activity, the end-of-day multiple-choice questions, is divided into three categories that test both egocentric and allocentric spatial knowledge ${ }^{39-42}$ : (1) landmark sequencing (eg, "You're walking along X St. from Y St. to Z St. Which landmark do you most closely pass?"); (2) distance judgement (eg, "Which landmark is closer to X?"); and (3) vector mapping (eg, "Facing X St. from $Y$, which degree represents the direction of Z?").

Finally, the third activity, the map-placement task, involves presenting two reproductions of the study map stripped of all information. Letters and numbers representing landmarks and streets, respectively, need to be matched to a list of learnt landmark and street names.

To increase compliance and engagement, in addition to earning potential coffee cards, auditory and written rewards are provided throughout training. Auditory rewards are presented in the form of short audio clips about each new landmark, with information about the landmark that is part of a greater fictional narrative created for each city. Written rewards are in the form of eight different encouraging pop-up messages appearing randomly throughout the week (eg, "Good work, keep it up!"). Placement of written and coffee card rewards are based on four randomised schedules, with a maximum of four combined rewards, at approximately $25 \%, 50 \%$, $75 \%$ and $100 \%$ of the way into the day. The four schedules were additionally randomised and each set as reward schedules for four different weeks.

\section{Active control condition protocol}

Participants randomly assigned to the active control condition complete a 16-week educational video intervention at the same intensity as the navigation training, also on a designated website hosted on the secure UHN server. Here, training involves watching videos on educational topics (ie, TED Talks), to control for placebo effects and for the effects of generalised environmental enrichment of the same dose as the targeted navigation training. For each day of training, participants are asked to select one of two possible videos three times, watching a total of three videos.

During protocol development, videos were selected by two raters, basing their decisions on the educational 
and engagement level. The videos were further screened for coarse language, sexual content, and highly political, religious or polarising topics. Any videos involving topics revolving around health, which could be regarded as providing medical advice, were also excluded (refer to online supplemental table 4 for the final list of videos).

Compliance is indexed by the amount of time patients spend on each of the videos. As an indirect measure, this is acknowledged as a potential limitation, but allows some insight into degree of compliance. To encourage maximal attention to the videos, at the end of each video, participants rate six aspects of the content (relevance, interest, comprehensibility) and speaker (quality of delivery, enthusiam, entertainment quality), on a scale of 1 (lowest) to 5 (highest). This rating task was chosen because it does not have a strong memory component associated with it and thus ensures the task is not targeting memory functioning. As an innate characteristic of self-administered therapy and generalised questions, attention is not able to be measured directly and is acknowledged as a potential limitation.

Additionally, as with navigation participants, they are given written rewards of encouragement with the same randomised placement described above.

\section{Outcome measures}

Table 1 summarises the timeline of the collection of each of the measures throughout the duration of the study, with detailed descriptions below.

\section{Spatial and memory measures (Objective 1a)}

The primary cognitive outcome measures include neartransfer, medium-transfer and far-transfer performancebased measures plus additional patient-reported outcomes. Near-transfer outcome measures (navigation intervention group only) include the following

\begin{tabular}{|c|c|c|c|}
\hline Phase & Timeline & Outcome category & Outcome measures \\
\hline \multirow[t]{6}{*}{ Pre-intervention } & Week 0 day 1 & Primary (imaging) & MRI \\
\hline & & Primary (experimental) & Day 1 medium-transfer tasks* \\
\hline & & Primary (clinical) & Clinical measures $\dagger$ \\
\hline & & Control & Demographics, mood $\ddagger$ \\
\hline & Week 0 day 2 & Primary (experimental) & $\begin{array}{l}\text { Day } 2 \text { medium-transfer tasks } \S \\
\text { Far-transfer tasks } \emptyset\end{array}$ \\
\hline & & Control & Physical activity** \\
\hline \multirow[t]{3}{*}{ Intervention } & Weeks $1-16$, days $1-5$ & Primary (experimental) & Near-transfer tasks †† \\
\hline & & Secondary (feasibility) & Compliance to intervention $\ddagger \ddagger$ \\
\hline & Weeks $1-16$, day 5 only & Secondary (feasibility) & How Much Is Too Much Scale \\
\hline \multirow[t]{7}{*}{ Post-intervention } & Week 17 day 1 & Primary (imaging) & MRI \\
\hline & & Primary (experimental) & Day 1 medium-transfer tasks* \\
\hline & & Primary (clinical) & Clinical measures $†$ \\
\hline & & Control & Physical activity ${ }^{\star \star}$ \\
\hline & Week 17 day 2 & Primary (experimental) & $\begin{array}{l}\text { Day } 2 \text { medium-transfer tasks } \S \\
\text { Far-transfer tasks } \emptyset\end{array}$ \\
\hline & & Secondary (feasibility) & Semistructured interview \\
\hline & Week 17 days $1-5$ & Primary (experimental) & Near-transfer tasksł‡ \\
\hline
\end{tabular}

*Tasks include Different Approach Task, Path Integration Task.

†Digit Span, Visual Spatial Span, SART, Symbol Digit Modalities Test, flanker inhibitory control and attention test, dimensional change card sort test, pattern comparison processing speed test, picture sequence memory test, RAVLT, RVDLT, GOSE.

$\ddagger$ Age, sex, injury severity, hours of therapy, BAI, BDI.

§CMFT, SBSOD, NSQ.

ПMIC, MST, EMQ.

**IPAQ.

††Training-related outcome measures, and comparison of performance on week 1 and untrained city (week 17) for navigation participants only.

¥¥Whether at least $80 \%$ of intervention has been completed, and average percentage of daily tasks completed.

BAI, Beck Anxiety Inventory; BDI, Beck Depression Inventory; CMFT, Cognitive Map Formation Test; EMQ, Everyday Memory Questionnaire; GOSE, Glasgow Outcome Scale Extended; IPAQ, International Physical Activity Questionnaire; MIC, Memory Image Completion Task; MST, Mnemonic Similarity Test; NSQ, Navigational Strategies Questionnaire; RAVLT, Rey Auditory Verbal Learning Test; RVDLT, Rey Visual Design Learning Test; SART, Sustained Attention to Response Test; SBSOD, Santa Barbara Sense of Direction Scale. 
within-task intervention components: (1) spatial learning ability based on end-of-day questions; (2) overall spatial ability improvement based on the difference in performance of end-of-week end-of-day questions, between earlier to later weeks of training; (3) independent navigation ability improvement on GSV-only reverse, alternate and blocked routes, based on differences in performance between earlier to later weeks of training; and (4) cognitive map formation ability, based on differences in endof-week performance between earlier and later weeks of training. Because the complexity of the cities increases throughout the 16 weeks of training, and participants may switch challenge levels, this is not a direct comparison of pre-intervention and post-intervention ability. Therefore, performance is also compared between the first week of training and an additional week in a city on which participants were not trained, following the completion of the intervention.

Medium-transfer measures are used to assess changes to spatial abilities that were not trained: (1) Cognitive Map Formation Test (CMFT; earlier version described in $\left.^{43}\right)$; (2) Different Approach Task ${ }^{44}$; and (3) Path Integration Task. ${ }^{45}$ Far-transfer measures include memory tasks sensitive to HPC integrity to assess generalisability of training to HPC-dependent abilities that were not directly trained: (1) Memory Image Completion Task $(\mathrm{MIC})^{46}{ }^{47}$; and (2) Mnemonic Similarity Test (MST) ${ }^{48}$ Patient-reported outcome measures include subjective changes to navigation and memory in daily life with the following self-report measures: (1) Santa Barbara Sense of Direction Scale (SBSOD) ${ }^{49}$; (2) Navigational Strategies Questionnaire (NSQ) ${ }^{50}$ and (3) Everyday Memory Questionnaire (EMQ). ${ }^{51}$

Alternate forms of the primary tasks are used (ie, MST, MIC, CMFT, Different Approach Task, Path Integration Task), with the specific form used for the pre-intervention and post-intervention assessments counterbalanced across participants. To maintain consistency in administration, data collection and data entry, experimenters undergo matched training and use scripts and detailed instructions. Coding of the key components of the measures are entered and checked by multiple experimenters.

\section{Neuropsychological battery (characterisation and additional outcomes)}

A comprehensive clinical neuropsychological assessment battery validated for use with individuals with TBI is administered by a trained neuropsychologist blind to group allocation, to characterise the participants at baseline and permit exploration of changes to performance following the intervention. The performance-based outcomes that are administered include the: (1) Wechsler Test of Adult Reading ${ }^{52}$ (for characterisation only); (2) Digit Span forwards and backwards ${ }^{53}$; Visual Spatial Span (forwards and backwards); (3) Rey Auditory Verbal Learning Test (RAVLT) ${ }^{54}$; (4) Rey Visual Design Learning Test (RVDLT) $)^{55}$; (5) Sustained Attention to Response Test $(\text { SART })^{56}$; (6) Symbol Digit Modalities Test ${ }^{57}$; and
(7) select subtests of the NIH toolbox, ${ }^{58}$ including the picture sequence memory test, flanker inhibitory control and attention test, dimensional change card sort test and pattern comparison processing speed test. Alternate forms of all tasks are employed as required. As a broader index of functional outcome, the Glasgow Outcome Scale Extended $^{59}$ is administered pre-intervention and postintervention, with a small subset of participants that will not have completed this outcome as it was added shortly after recruitment began. In addition, mood assessments are employed, including the Beck Depression Inventory-II (BDI-II) and the Beck Anxiety Inventory (BAI)..$^{60} 61$

For each participant, clinical feedback is provided via phone call by a neuropsychologist, to the participant and their significant other or caregiver. Information from their clinical interview, performance across the clinical measures and medical chart is integrated. Feedback includes: (1) a review of the purpose and expectations of the assessment; (2) a review of strengths and weaknesses across cognitive domains that have been observed, and connecting them (if applicable) to clinical complaints or symptoms; (3) a discussion regarding personal goals and how best to use identified strengths to offset their weaknesses; and (4) further referrals to medical professionals, if applicable.

\section{Control variables}

Demographic information, injury history and hours of therapy for all participants are collected using a secure, online survey prior to the pre-intervention assessment. At the pre-intervention assessment, a personal interview is conducted by a neuropsychologist to validate the contents of the survey and gather additional information. In addition to inclusion for clinical feedback, BAI and BDI-II scores are also considered as control variables. ${ }^{60} 61$ Participants' current levels of physical activity are assessed using the International Physical Activity QuestionnaireShort Form (IPAQ). ${ }^{62}$

\section{Structural MRI of the HPC (Objective 1b)}

A Siemens Prisma 3 Tesla scanner with a 32-channel head coil is used for imaging acquisition. The primary imaging measures include total HPC volumes and HPC substructures. The secondary imaging measures include diffusion tensor imaging (DTI) to examine the integrity of WM tracts involving the HPC (ie, fimbria-fornix pathway). The full list of MRI sequences can be found in online supplemental material 1 .

\section{Feasibility measures (Objective 2)}

General feasibility of the intervention is determined by the recruitment rate, retention rate (including factors influencing retention) and compliance rates, based on objective rates and subjectively through daily, weekly and biweekly self-reported questionnaires. Recruitment rate is determined by dividing the number of participants consented by the number of eligible participants approached, retention rate is determined by dividing the number of consented participants at baseline by the 
number of consented participants retained at follow-up, and compliance rate is determined by calculating the percentage of participants adhering to at least $80 \%$ of the training protocol, as well as by calculating the average of each individual participant's percentage of completion of each of the daily tasks across the entire duration of the intervention. Retention and compliance rates close to $100 \%$ are desired, but $70 \%$ or greater are considered acceptable, based on previous studies assessing feasibility of self-administered computerised interventions in this type of population. ${ }^{63-65}$

The degree of burden to the participants in completing the intervention is assessed using the How Much Is Too Much Scale, ${ }^{33}$ administered each week to measure physical, mental and mood symptom onset following intervention participation, incorporated on the intervention website at the end of the week's tasks. Participants placed in the targeted navigation intervention group also complete a semistructured interview following all of the outcome measures. The experimenter who administers the remote assessments conducts the interview over the phone by following the interview guide (online supplemental table 2). Each interview lasts approximately $30 \mathrm{~min}$, is digitally recorded, then transcribed verbatim for data analysis. Participants are asked about their experiences with the intervention, uptake of web-based technology, perceived barriers and facilitators to participating, as well as strengths, weaknesses and impact of the programme on their daily life. ${ }^{66}$

\section{STATISTICAL PROCEDURES}

\section{Objectives $1 \mathrm{a}$ and $1 \mathrm{~b}$ (efficacy)}

To address Objective 1a (efficacy for behavioural outcomes), descriptive and inferential statistics will be employed to examine group-level changes in the following cognitive outcomes (experimental and clinical): (1) fartransfer tasks associated with executive functioning (Digit Span, Visual Spatial Span, SART, Symbol Digit Modalities Test, flanker inhibitory control and attention test, dimensional change card sort test, pattern comparison processing speed test); (2) far-transfer tasks and subjective measures associated with memory (MIC, MST, RAVLT, RVDLT, picture sequence memory test, EMQ); and (3) medium-transfer tasks and subjective measures associated with spatial abilities (CMFT, Different Approach Task, Path Integration Task, SBSOD, NSQ).

A principal component analysis will be conducted for dimension reduction of these measures, and the resultant components will be used as predictors in a series of between-group (targeted navigation and active control) analyses, which will be used to estimate the average causal treatment effect. Secondary control measures will then be introduced individually to test for interactions that, if present, indicate that the corresponding variable acts as a moderator of the treatment effect, including depression (BDI-II), anxiety (BAI), age, sex, injury severity (ie,
PTA), hours of therapy and physical activity (IPAQ). If so, specific effects will be estimated.

Within the targeted navigation group, changes to neartransfer outcomes will be assessed using single-group analyses on the training-related outcome measures. The analyses will be carried out with mixed models using information available from all participants, including those with partially missing data (combining mixed models with multiple imputation for longitudinal data) to reduce potential bias due to attrition. Analyses will be carried out 'as randomised' (intent-to-treat analyses) where possible, as well as 'per protocol' taking the degree of compliance into account. We anticipate group effects and group by time interactions, with improvements in the navigation group at post-intervention (vs control). For navigation participants, we also anticipate improvements on the training-related outcome measures.

For Objective 1b (efficacy of the intervention to reduce HPC degeneration), the same analyses described above for the cognitive measures will be undertaken for our imaging outcomes. Additional HPC subfield analyses will be completed based on manual segmentation of the T2 volumes, where data analysts will be blinded to the allocation of the participant. We expect the dentate gyrus and CA1 subregions to most strongly express degeneration and preservation trajectories due to roles in allocentric navigation and neurogenesis. For DTI, we expect an offset of previously reported fornix degeneration following navigation training. ${ }^{7}$ Cingulum integrity could also be expected to be modulated with improved HPC function and/or structure.

\section{Objective 2 (feasibility)}

This objective will be achieved by measuring recruitment and retention, and compliance in (i) completion of the interventions, and (ii) completion of all behavioural outcome measures of the study. Acceptability/tolerance of procedures is measured with the How Much Is Too Much Scale. A semistructured interview will help identify barriers to completion and elicit suggestions for improvement. Descriptive statistics will be used to summarise the outcomes of the feasibility component of the study.

Inductive manual thematic analysis ${ }^{67}$ will be conducted on the recorded responses to the questions from the semistructured interviews to assess the themes, staying close to participants' own words. ${ }^{689}$ A subset of interview transcripts will be initially coded by two experimenters to ensure agreement and standardisation of a coding framework to be applied to the remaining transcripts. Following this, the remainder of transcripts will be coded independently by each experimenter. On completion, reviewers will meet to reconcile coding of major identifiable themes of each transcript, allowing for enhanced reflexivity and rigour.

To aid in the organisation of data and visualisation of emergent themes (eg, word frequency query, mind map), coding notes will be entered into $\mathrm{NVivo}^{70}$ and clustered into groups and categories. To maximise credibility and 
impartiality, the broader research team will meet to discuss the developing analysis, where new themes may be considered, and until consensus is reached and theme labels are agreed on.

\section{ETHICS AND DISSEMINATION}

This study has been approved by the UHN Research Ethics Board and the Research Oversight and Compliance Office at the University of Toronto. This study is conducted in accordance with the Declaration of Helsinki and the Tri-Council Policy Statement: Ethical Conduct for Research Involving Humans, second edition. ${ }^{71}$ The present study began on 20 October 2018 and recruitment is ongoing. Any modifications to the present protocol will be submitted as formal amendments to the original ethics application and reviewed by the above ethics boards prior to their implementation. All participants are recruited through an informed consent protocol in-person and by telephone (please see online supplemental material 2 for a model consent form). At the time of consent, participants are assured that withdrawing from the study will not affect the care they receive at Toronto Rehabilitation Institute. Participants are informed that the study involves minimal risks (ie, fatigue from assessments or intervention, claustrophobia during MRI). Participants may leave the study at any time.

The protocol follows the guidelines prescribed by the Standard Protocol Items: Recommendations for Interventional Trials Statement. ${ }^{72}$ All collected physical data are stored securely in locked cabinets, and electronic data are stored on a secure server at Toronto Rehabilitation Institute. Only the direct study team will have access to identifying information, which is kept confidential, and to the final dataset. Results summarising the anonymised data will be presented at academic and clinical conferences and findings will be submitted to peer-reviewed journals. Authorship for publications will be determined based on the uniform requirements for manuscripts submitted to biomedical journals. ${ }^{73}$ Key findings will be shared directly with participants who have participated in the study by email, summarising the broad results in a simplified and accessible format, and will be made available to the public through media releases shared through the Marketing and Communications departments at Toronto Rehabilitation Institute and the Rotman Research Institute at Baycrest.

\section{SIGNIFICANCE AND IMPACT}

The strength and novelty of the present study is the unique design of an intervention that can be completed by participants in their own homes using widely available software, which can therefore be delivered with minimal resources, anywhere in the world. This enhances the scalability and reach of the intervention, allowing the study to be replicated and the intervention to be available to large samples of patients with TBI and other patient populations with similar neuropathology. Furthermore, using the principles of $\mathrm{EE}$, the intervention is designed to be continuously novel and challenging, allowing participants to remain engaged while stimulating the HPC through tasks in which it is known to be involved. Additionally, the study employs an active control to allow more robust conclusions to be made regarding the effectiveness of a targeted navigation intervention for m-sTBI, compared with generalised EE.

Finally, the online setting of the intervention allows for real-time data collection to monitor compliance and performance while maintaining accurate records. Importantly, the proposed research aims to develop infrastructure for ongoing neurorehabilitation in remote communities and for individuals who may face other barriers to obtaining necessary resources (eg, reduced mobility or financial burden). The self-administered feasibility questionnaire as well as the post-intervention interview will help to ascertain barriers and facilitators to retention and compliance for our intervention, with relevance to other computerised cognitive interventions for individuals with m-sTBI, to support the expansion of treatment options for individuals in this population.

\section{Author affiliations}

${ }^{1}$ Department of Psychology, University of Toronto, Toronto, Ontario, Canada

${ }^{2}$ Rotman Research Institute at Baycrest, Toronto, Ontario, Canada

${ }^{3} \mathrm{KITE}$, Toronto Rehabilitation Institute, Toronto, Ontario, Canada

${ }^{4}$ Graduate Department of Rehabilitation Science, University of Toronto, Toronto, Ontario, Canada

${ }^{5}$ Department of Psychology, The State University of New York, Geneseo, New York, USA

${ }^{6}$ Department of Computing and Software, McMaster University, Hamilton, Ontario, Canada

${ }^{7}$ Department of Psychology, York University, Toronto, Ontario, Canada

${ }^{8} \mathrm{Global}$ Health Program, Faculty of Health Sciences, McMaster University, Hamilton, Ontario, Canada

${ }^{9}$ Department of Psychiatry, University of Toronto, Toronto, Ontario, Canada

Twitter Zorry Belchev @z_belchev, Mary Ellene Boulos @marymeetsworld and Michael Johnathan Charles Bray @TheNeurocyclist

Acknowledgements We would like to thank all of the volunteers, research assistants and undergraduate project students who graciously offered their time to assist in the development of the intervention: Taha Arshad, Marta Bogacki, Priyanka Prince, Sonia Persaud, Michelle Gomez, Robert Dydynsky, Alexander Drohobycky, Mikael Salnikov, Reid Syrydiuk, Ginelle Feliciano, Roy Kuo, Maleeha Khan, Gina D'Souza, Mubina Butt, Samreen Aziz and Madison Fraser. Finally, we would also like to acknowledge our recruitment contacts and partners who are central in strengthening our recruitment reach in order to assess the feasibility and efficacy of this intervention in patients: Dr Matthew Burke (Sunnybrook Health Sciences Center), Dr Karl F Gunnarsson (West Park Healthcare Centre), Kamilah Francis and Crystal McCollum (March of Dimes Canada), and Michelle Pangilinan (Community Brain Injury Services).

Contributors $A G$ and $R G$ conceived the original idea for the intervention, with $B C$ as study manager. $A G$ and $R G$ are also supervising the study, are helping maintain recruitment avenues and contributed to the final manuscript. ZB developed the materials for the intervention with assistance and supervision by $A G$ and $R G$, and with the aid of AL and AC. ZB, MEB and JR all contributed to the implementation of the intervention on the training website, with $\mathrm{JO}$ completing the initial methodology for GSV and overseeing ND as programmer, and with assistance from TW and AL. MJCB and MEB developed the semistructured interview that will be administered to patients following completion of the study. $\mathrm{KJ}$ oversees the recruitment and screening of patients, with help from EJ, JR, MEB and ZB, as well as all other administrative aspects of the study. ZB, JR and MEB oversee data collection and 
training patients on the intervention, with EJ completing the administration of neuropsychological assessments and reports, and TW assisting in data collection and training. ZB, MEB and JR wrote the manuscript with consultation from $A G$ and $\mathrm{RG}$

Funding This work is supported and funded by the Ontario Neurotrauma Foundation (ONF; 2017-ABI-INFRA-1035), the Canadian Centre for Aging \& Brain Health Innovation (CABHI), the Canadian Traumatic Brain Injury Research Consortium (CTRC), the Branch Out Neurological Foundation and the Canada Research Chairs (950-230647).

Disclaimer None of the funding sources had any role in the design of the protocol nor will be involved in its execution, analyses, data interpretation or dissemination of results.

Competing interests None declared.

Patient consent for publication Not required.

Provenance and peer review Not commissioned; externally peer reviewed.

Supplemental material This content has been supplied by the author(s). It has not been vetted by BMJ Publishing Group Limited (BMJ) and may not have been peer-reviewed. Any opinions or recommendations discussed are solely those of the author(s) and are not endorsed by BMJ. BMJ disclaims all liability and responsibility arising from any reliance placed on the content. Where the content includes any translated material, BMJ does not warrant the accuracy and reliability of the translations (including but not limited to local regulations, clinical guidelines, terminology, drug names and drug dosages), and is not responsible for any error and/or omissions arising from translation and adaptation or otherwise.

Open access This is an open access article distributed in accordance with the Creative Commons Attribution Non Commercial (CC BY-NC 4.0) license, which permits others to distribute, remix, adapt, build upon this work non-commercially, and license their derivative works on different terms, provided the original work is properly cited, appropriate credit is given, any changes made indicated, and the use is non-commercial. See: http://creativecommons.org/licenses/by-nc/4.0/.

\section{ORCID iDs}

Zorry Belchev http://orcid.org/0000-0002-9361-0865

Mary Ellene Boulos http://orcid.org/0000-0001-5046-598X

Julia Rybkina http://orcid.org/0000-0001-5996-3512

Michael Johnathan Charles Bray http://orcid.org/0000-0003-4650-1313

\section{REFERENCES}

1 World Health Organization. World health statistics 2015, 2015. Available: https://www.who.int/gho/publications/world_health_ statistics/2015/en/

2 Cole JH, Jolly A, de Simoni S, et al. Spatial patterns of progressive brain volume loss after moderate-severe traumatic brain injury. Brain 2018;141:822-36.

3 Green REA, Colella B, Maller JJ, et al. Scale and pattern of atrophy in the chronic stages of moderate-severe TBI. Front Hum Neurosci 2014;8:67.

$4 \mathrm{Ng} \mathrm{K}$, Mikulis DJ, Glazer J, et al. Magnetic resonance imaging evidence of progression of subacute brain atrophy in moderate to severe traumatic brain injury. Arch Phys Med Rehabil 2008;89:S35-44.

5 Green REA. Editorial: brain injury as a neurodegenerative disorder. Front Hum Neurosci 2015;9:615.

6 Masel BE, DeWitt DS. Traumatic brain injury: a disease process, not an event. J Neurotrauma 2010;27:1529-40.

7 Adnan A, Crawley A, Mikulis D, et al. Moderate-severe traumatic brain injury causes delayed loss of white matter integrity: evidence of fornix deterioration in the chronic stage of injury. Brain Inj 2013;27:1415-22.

8 McCarthy MM. Stretching the truth. Why hippocampal neurons are so vulnerable following traumatic brain injury. Exp Neurol 2003;184:40-3.

9 Mckee AC, Daneshvar DH. The neuropathology of traumatic brain injury. Handb Clin Neurol 2015;127:45-66.

10 Graham DI. Adams \& Graham's Introduction to Neuropathology 3ed. In: Nicoll JAR, Bone I, Graham DI, eds. Adams \& Graham's Introduction to Neuropathology 3ed. Boca Raton, Florida: CRC Press, 2006.

11 Smith DH, Hicks R, Povlishock JT. Therapy development for diffuse axonal injury. J Neurotrauma 2013;30:307-23.
12 Skelton RW, Ross SP, Nerad L, et al. Human spatial navigation deficits after traumatic brain injury shown in the arena maze, a virtual Morris water maze. Brain Inj 2006;20:189-203.

13 Kersel DA, Marsh NV, Havill JH, et al. Neuropsychological functioning during the year following severe traumatic brain injury. Brain Inj 2001;15:283-96.

14 Zec RF, Zellers D, Belman J, et al. Long-term consequences of severe closed head injury on episodic memory. J Clin Exp Neuropsychol 2001;23:671-91.

15 Bigler ED, Johnson SC, Anderson CV, et al. Traumatic brain injury and memory: the role of hippocampal atrophy. Neuropsychology 1996;10:333-42.

16 Changoor A, Colella B, Monette G, et al. Moderate-Severe TBI as a progressive disorder: cognitive declines in the chronic stages of injury. Neurology 2020;94 https://n.neurology.org/content/94/15 Supplement/5035.abstract

17 Ledig C, Kamnitsas K, Koikkalainen J, et al. Regional brain morphometry in patients with traumatic brain injury based on acute- and chronic-phase magnetic resonance imaging. PLoS One 2017;12:e0188152.

18 Bramlett HM, Dietrich WD, Dalton Dietrich W. Long-term consequences of traumatic brain injury: current status of potential mechanisms of injury and neurological outcomes. J Neurotrauma 2015;32:1834-48.

19 Jullienne A, Obenaus A, Ichkova A, et al. Chronic cerebrovascular dysfunction after traumatic brain injury. J Neurosci Res 2016;94:609-22.

20 Tomaszczyk JC, Green NL, Frasca D, et al. Negative neuroplasticity in chronic traumatic brain injury and implications for neurorehabilitation. Neuropsychol Rev 2014;24:409-27.

21 Mahncke HW, Bronstone A, Merzenich MM. Brain plasticity and functional losses in the aged: scientific bases for a novel intervention. Prog Brain Res 2006;157:81-109.

22 Aimone JB, Li Y, Lee SW, et al. Regulation and function of adult neurogenesis: from genes to cognition. Physiol Rev 2014;94:991-1026.

23 Boldrini M, Fulmore CA, Tartt AN, et al. Human hippocampal neurogenesis persists throughout aging. Cell Stem Cell 2018;22:589-99.

24 Moreno-Jiménez EP, Flor-García M, Terreros-Roncal J, et al. Adult hippocampal neurogenesis is abundant in neurologically healthy subjects and drops sharply in patients with Alzheimer's disease. Nat Med 2019;25:554-60.

25 Scholz J, Allemang-Grand R, Dazai J, et al. Environmental enrichment is associated with rapid volumetric brain changes in adult mice. Neuroimage 2015;109:190-8.

26 Konishi K, Bohbot VD. Spatial navigational strategies correlate with gray matter in the hippocampus of healthy older adults tested in a virtual maze. Front Aging Neurosci 2013;5:1.

27 Lithfous S, Dufour A, Després O. Spatial navigation in normal aging and the prodromal stage of Alzheimer's disease: insights from imaging and behavioral studies. Ageing Res Rev 2013;12:201-13.

28 West GL, Konishi K, Bohbot VD. Video games and hippocampusdependent learning. Curr Dir Psychol Sci 2017;26:152-8.

29 Lövdén M, Schaefer S, Noack H, et al. Spatial navigation training protects the hippocampus against age-related changes during early and late adulthood. Neurobiol Aging 2012;33:620.e9-620. e22.

30 Lövdén M, Schaefer S, Noack H, et al. Performance-related increases in hippocampal N-acetylaspartate (NAA) induced by spatial navigation training are restricted to BDNF Val homozygotes. Cereb Cortex 2011;21:1435-42.

31 Faul F, Erdfelder E, Buchner A, et al. Statistical power analyses using $\mathrm{G}^{*}$ Power 3.1: tests for correlation and regression analyses. Behav Res Methods 2009;41:1149-60.

32 Raz N, Ghisletta P, Rodrigue KM, et al. Trajectories of brain aging in middle-aged and older adults: regional and individual differences. Neuroimage 2010;51:501-11.

33 Tomaszczyk J, Sharma B, Chan AA, et al. Measuring cognitive assessment and intervention burden in patients with acquired brain injured: development of the "how much is too much" questionnaire. $J$ Rehabil Med 2018;50:519-26.

34 laria G, Petrides M, Dagher A, et al. Cognitive strategies dependent on the hippocampus and caudate nucleus in human navigation: variability and change with practice. J Neurosci 2003;23:5945-52.

35 Ohnishi T, Matsuda H, Hirakata M, et al. Navigation ability dependent neural activation in the human brain: an fMRI study. Neurosci Res 2006;55:361-9.

36 O'Keefe J, Dostrovsky J. The hippocampus as a spatial map. Preliminary evidence from unit activity in the freely-moving rat. Brain Res 1971;34:171-5. 
37 Bohbot VD, Lerch J, Thorndycraft B, et al. Gray matter differences correlate with spontaneous strategies in a human virtual navigation task. J Neurosci 2007;27:10078-83.

38 Howard LR, Javadi AH, Yu Y, et al. The hippocampus and entorhinal cortex encode the path and Euclidean distances to goals during navigation. Curr Biol 2014;24:1331-40.

39 Hirshhorn M, Grady C, Rosenbaum RS, et al. The hippocampus is involved in mental navigation for a recently learned, but not a highly familiar environment: a longitudinal fMRI study. Hippocampus 2012;22:842-52.

40 Rosenbaum RS, Ziegler M, Winocur G, et al. "I have often walked down this street before": fMRI studies on the hippocampus and other structures during mental navigation of an old environment. Hippocampus 2004;14:826-35.

41 Rosenbaum RS, Cassidy BN, Herdman KA. Patterns of preserved and impaired spatial memory in a case of developmental amnesia. Front Hum Neurosci 2015;9:196.

42 Waller D, Hodgson E. Transient and enduring spatial representations under disorientation and self-rotation. J Exp Psychol Learn Mem Cogn 2006;32:867-82

43 Corrow JC, Corrow SL, Lee E, et al. Getting lost: topographic skills in acquired and developmental prosopagnosia. Cortex 2016;76:89-103.

44 Wiener JM, Carroll D, Moeller S, et al. A novel virtual-reality-based route-learning test suite: assessing the effects of cognitive aging on navigation. Behav Res Methods 2020;52:630-40.

45 Persson J, Herlitz A, Engman J, et al. Remembering our origin: gender differences in spatial memory are reflected in gender differences in hippocampal lateralization. Behav Brain Res 2013;256:219-28.

46 Vieweg P, Stangl M, Howard LR, et al. Changes in pattern completion - a key mechanism to explain age-related recognition memory deficits? Cortex 2015;64:343-51.

47 Vieweg P, Riemer M, Berron D, et al. Memory image completion: establishing a task to behaviorally assess pattern completion in humans. Hippocampus 2019;29:340-51.

48 Stark SM, Yassa MA, Lacy JW, et al. A task to assess behavioral pattern separation (BPs) in humans: data from healthy aging and mild cognitive impairment. Neuropsychologia 2013;51:2442-9.

49 Hegarty Met al. Development of a self-report measure of environmental spatial ability. Intelligence 2002;30:425-47.

50 Brunec IK, Bellana B, Ozubko JD, et al. Multiple scales of representation along the hippocampal anteroposterior axis in humans. Curr Biol 2018;28:2129-35.

51 Sunderland A, Harris JE, Baddeley AD. Do laboratory tests predict everyday memory? A neuropsychological study. J Verbal Learning Verbal Behav 1983;22:341-57.

52 Wechsler D. Wechsler test of adult reading (WTAR). San Antonio, TX: The Psychological Corporation, 2001.

53 Wechsler D. Manual for the wechsler adult intelligence scale. New York: Psychological Corp, 1981

54 Rey A. L'examen clinique en psychologie. 2 ed. Paris: Presses universitaires de France, 1959.

55 Rey A. L'examen clinique en psychologie. 1 éd. Paris: Presses universitaires de France, 1958.
56 Robertson IH, Manly T, Andrade J, et al. 'Oops!': performance correlates of everyday attentional failures in traumatic brain injured and normal subjects. Neuropsychologia 1997;35:747-58.

57 Smith A. Symbol digits modalities test. Los Angeles: Western Psychological Sciences, 1982.

58 Gershon RC, Cella D, Fox NA, et al. Assessment of neurological and behavioural function: the NIH Toolbox. Lancet Neurol 2010;9:138-9.

59 Wilson JT, Pettigrew LE, Teasdale GM. Structured interviews for the Glasgow outcome scale and the extended Glasgow outcome scale: guidelines for their use. J Neurotrauma 1998;15:573-85.

60 Beck AT, Epstein N, Brown G, et al. An inventory for measuring clinical anxiety: psychometric properties. J Consult Clin Psychol 1988;56:893-7.

61 Beck AT, Steer RA, Brown GK. Manual for the Beck depression inventory-II. San Antonio, TX: Psychol Corp, 1996: 1-82.

62 Craig CL, Marshall AL, Sjöström M, et al. International physical activity questionnaire: 12-country reliability and validity. Med Sci Sports Exerc 2003;35:1381-95.

63 Lebowitz MS, Dams-O'Connor K, Cantor JB. Feasibility of computerized brain plasticity-based cognitive training after traumatic brain injury. J Rehabil Res Dev 2012;49:1547.

64 O'Neil-Pirozzi TM, Hsu H. Feasibility and benefits of computerized cognitive exercise to adults with chronic moderate-to-severe cognitive impairments following an acquired brain injury: a pilot study. Brain Inj 2016;30:1617-25.

65 Sharma B, Tomaszczyk JC, Dawson D, et al. Feasibility of online self-administered cognitive training in moderate-severe brain injury. Disabil Rehabil 2017;39:1380-90.

66 Cresswell JW, Plano-Clark VL, Gutmann ML. Advanced mixed methods research designs. In: Tashakkori A, Teddlie C, eds. Handbook of mixed methods in social and behavioral research Thousand Oaks: Sage, 2003: 209-40.

67 Braun V, Clarke V. Using thematic analysis in psychology. Qual Res Psychol 2006;3:77-101.

68 Sandelowski M. Whatever happened to qualitative description? Res Nurs Health 2000;23:334-40.

69 Sandelowski M. What's in a name? Qualitative description revisited. Res Nurs Health 2010;33:77-84

70 International Q. NVivo qualitative data analysis software, 1999. Available: https://www.qsrinternational.com/nvivo-qualitative-dataanalysis-software/home

71 Canadian Institutes of Health Research, Natural Sciences and Engineering Research Council of Canada, Social Sciences and Humanities Research Council. Tri-council policy statement: ethical conduct for research involving humans 2018 https://ethics.gc.ca/ eng/policy-politique_tcps2-eptc2_2018.html

72 Chan A-W, Tetzlaff JM, Altman DG, et al. Spirit 2013 statement: defining standard protocol items for clinical trials. Ann Intern Med 2013:158:200-7.

73 International Committee of Medical Journal Editors. Uniform requirements for manuscripts submitted to biomedical journals: writing and editing for biomedical publication, 2010. Available: www. ICMJE.org 